\title{
Thin-shell wormholes supported by total normal matter
}

\author{
S. Habib Mazharimousavi ${ }^{\mathrm{a}}$, M. Halilsoy ${ }^{\mathrm{b}}$ \\ Department of Physics, Eastern Mediterranean University, Gazimağusa, Turkey
}

Received: 11 March 2014 / Accepted: 31 August 2014 / Published online: 23 September 2014

(C) The Author(s) 2014. This article is published with open access at Springerlink.com

\begin{abstract}
The Zipoy-Voorhees-Weyl (ZVW) spacetime characterized by mass $(M)$ and oblateness $(\delta)$ is proposed in the construction of viable thin-shell wormholes (TSWs). A departure from spherical/cylindrical symmetry yields a positive total energy in spite of the fact that the local energy density may take negative values. We show that oblateness of the bumpy sources/black holes can be incorporated as a new degree of freedom that may play a role in the resolution of the exotic matter problem in TSWs. A small velocity perturbation reveals, however, that the resulting TSW is unstable.
\end{abstract}

\section{Introduction}

Until popularized by Morris and Thorne [1] the idea of a spacetime wormhole introduced in the 1930s by Einstein and Rosen [2] was considered non-physical and largely was taken as a fantasy. Although all kinds of spherically/cylindrically symmetric metrics known to date were tried, the wormhole concept was shadowed by the required negative total energy. While it was easy to resort to a quantum field theoretical negative energy as a remedy, the absence of large scale quantum systems persisted as another serious handicap. For such reasons relying on classical physics and searching for support within this context seems indispensable. Even to minimize the negative (exotic) energy the idea of a thin-shell wormhole (TSW) was developed (see [3-27] and references cited therein). By construction, in all these studies the otherwise non-traversable wormhole throat that connects two different universes has a circular topology.

In this study we add oblateness as a new degree of freedom represented by the parameter $\delta(-\infty<\delta<\infty)$ and show that for certain range of $\delta$ total energy becomes positive to

\footnotetext{
a e-mail: habib.mazhari@emu.edu.tr

b e-mail: mustafa.halilsoy@emu.edu.tr
}

avoid exotic sources. This happens in the Zipoy-VoorheesWeyl (ZVW) spacetime [28-30] with a quadrupole moment $Q=\frac{1}{3} M^{3} \delta\left(1-\delta^{2}\right)$, where $M$ is the mass of the bumpy object (or the black hole). Naturally for $\delta=1$ one recovers the spherical Schwarzschild geometry. It should be added that integrability and chaotic behavior of the ZVW spacetime still are not well understood [31,32]. An asymptotically flat, rotating ZVW metric was discovered by Tomimatsu and Sato (TS) $[33,34]$, which, similar to its static predecessor, remains from the physics stand point yet unclear. Once the problem of gravitational wave detection is overcome we expect that non-Kerr (i.e., $\delta \neq 1$ ) multipoles of the entire TS family can be detected. We note also that a non-asymptotically flat extension of the ZVW metric is also available whose physics is yet to be understood $[35,36]$. Herein we concentrate on the static ZVW metric in general relativity and construct a thin-shell wormhole (TSW) in this spacetime.

We should add that in the context of the modified theories of gravity, previously, there have been some attempts to introduce a thin-shell wormhole supported by positive/normal matter [37-44]. From this token it was realized that normal matter is possible only for the exotic branch solution of the Einstein-Gauss-Bonnet field equation [37-44].

The paper is organized as follows. The construction of TSW in ZVW spacetime is carried out in Sect. 2. Integration of the total energy is achieved in Sect. 3. A stability analysis follows in Sect. 4, and a Conclusion in Sect. 5 completes the paper.

\section{ZVW thin-shell wormhole (TSW)}

The two parameter ZVW spacetime in the prolate spheroidal coordinates is described by the line element

$$
\begin{aligned}
\mathrm{d} s^{2}= & -A(x) \mathrm{d} t^{2}+B(x, y) \mathrm{d} x^{2} \\
& +C(x, y) \mathrm{d} y^{2}+F(x, y) \mathrm{d} \varphi^{2}
\end{aligned}
$$


in which

$$
\begin{aligned}
& A=\left(\frac{x-1}{x+1}\right)^{\delta}, \quad B=\frac{k^{2}}{A}\left(\frac{x^{2}-1}{x^{2}-y^{2}}\right)^{\left(\delta^{2}-1\right)} \\
& C=B\left(\frac{x^{2}-1}{1-y^{2}}\right), \quad F=\frac{k^{2}}{A}\left(x^{2}-1\right)\left(1-y^{2}\right),
\end{aligned}
$$

where $k=\frac{M}{\delta}$ and the ranges of the coordinates are $1<x$, $0 \leq y^{2} \leq 1, \varphi \in[0,2 \pi]$ and $-\infty<t<\infty$. We note that $-\infty<\delta<\infty$ such that $\delta=0$ corresponds to a flat spacetime, and with $\delta=1$ one finds the Schwarzschild black hole solution with the horizon located at $x=1$. For the case $\delta \neq 1$ the hypersurface $x=1$ is a true curvature singularity (naked singularity) [45-49]. As we shall see, the asymptotic behavior of the ZV spacetime for $x \rightarrow \infty$ and $\delta>1$ is of our interest. It can be seen from (1) that, in the limit $x \rightarrow \infty$, it becomes

$\mathrm{d} s^{2}=-\mathrm{d} t^{2}+k^{2} \mathrm{~d} x^{2}+k^{2} x^{2}\left(\frac{\mathrm{d} y^{2}}{1-y^{2}}+\left(1-y^{2}\right) \mathrm{d} \varphi^{2}\right)$,

for which after the redefinition $k x=r$ and $y=\cos \theta$, the line element becomes

$\mathrm{d} s^{2}=-\mathrm{d} t^{2}+\mathrm{d} r^{2}+r^{2}\left(\mathrm{~d} \theta^{2}+\sin ^{2} \theta \mathrm{d} \varphi^{2}\right)$,

which is flat.

The construction of a thin-shell wormhole (TSW) follows the standard procedure of cutting and pasting [37-44]. We consider two copies of ZVW spacetime and we remove from each

$\mathcal{M}^{ \pm}=\left\{x^{ \pm}<a, \quad 1<a\right\}$

in which $a$ is constant outside the singularities/horizons. We should comment here that the minimality conditions of Hochberg and Visser [50], which are also known as the generalized flare-out conditions, in static wormholes do not apply in the present case of TSW [51]. At the throat two spacetimes are identified to make a complete manifold. We introduce next the induced coordinates $\xi^{i}=(\tau, y, \phi)$ on the wormhole's throat with its proper time $\tau$. The two coordinates are related by

$g_{i j}=\frac{\partial x^{\alpha}}{\partial \xi^{i}} \frac{\partial x^{\beta}}{\partial \xi^{j}} g_{\alpha \beta}$,

so that the induced metric on the throat $\Sigma$ reads

$g_{i j}=\operatorname{diag}[-1, C(a(\tau), y), F(a(\tau), y)]$.

The Israel junction conditions [52-56] on $\Sigma$ take the form $(c=8 \pi G=1)$

$$
\left\langle K_{i}^{j}\right\rangle-\langle K\rangle \delta_{i}^{j}=-S_{i}^{j},
$$

in which $\langle$.$\rangle stands for a jump across the hypersurface. K_{i}^{j}$ is the extrinsic curvature defined by

$K_{i j}^{( \pm)}=-n_{\gamma}^{( \pm)}\left(\frac{\partial^{2} x^{\gamma}}{\partial \xi^{i} \partial \xi^{j}}+\Gamma_{\alpha \beta}^{\gamma} \frac{\partial x^{\alpha}}{\partial \xi^{i}} \frac{\partial x^{\beta}}{\partial \xi^{j}}\right)_{\Sigma}$

with the normal unit vector

$n_{\gamma}^{( \pm)}=\left( \pm\left|g^{\alpha \beta} \frac{\partial \mathcal{H}}{\partial x^{\alpha}} \frac{\partial \mathcal{H}}{\partial x^{\beta}}\right|^{-1 / 2} \frac{\partial \mathcal{H}}{\partial x^{\gamma}}\right)$.

Note that $\langle K\rangle=\operatorname{Trace}\left\langle K_{i}^{j}\right\rangle$ and $S_{i}^{j}=\operatorname{diag}\left(-\sigma, P_{y}, P_{\phi}\right)$ is the energy-momentum tensor on the thin shell. The parametric equation of the hypersurface $\Sigma$ is given by

$\mathcal{H}(x, a(\tau))=x-a(\tau)=0$.

The normal unit vectors to $\mathcal{M}_{ \pm}$are found to be

$n_{\gamma}^{( \pm)}= \pm(-\sqrt{A B} \dot{a}, B \sqrt{\Delta}, 0,0)_{\Sigma}$

with $\Delta=\frac{1}{B}+\dot{a}^{2}$ and $\dot{a}=\frac{\mathrm{d} a}{\mathrm{~d} \tau}$. The resulting extrinsic curvature components are

$K_{\tau}^{\tau( \pm)}= \pm \frac{\ddot{a}+\left(\frac{B_{a}}{B}+\frac{A_{a}}{A}\right) \frac{\dot{a}^{2}}{2}+\frac{A_{a}}{2 A B}}{\sqrt{\Delta}}$

$K_{y}^{y( \pm)}= \pm \frac{C_{a}}{2 C} \sqrt{\Delta}$

$K_{\varphi}^{\varphi( \pm)}= \pm \frac{F_{a}}{2 F} \sqrt{\Delta}$

in which a subscript $a$ stands for $\frac{\partial}{\partial a}$. The surface energymomentum tensor has components defined by

$$
\begin{aligned}
\sigma & =-\left(\frac{C_{a}}{C}+\frac{F_{a}}{F}\right) \sqrt{\Delta} \\
P_{y} & =\frac{2 \ddot{a}+\left(\frac{B_{a}}{B}+\frac{A_{a}}{A}\right) \dot{a}^{2}+\frac{A_{a}}{A B}}{\sqrt{\Delta}}+\frac{\sqrt{\Delta} F_{a}}{F} \\
P_{\varphi} & =\frac{2 \ddot{a}+\left(\frac{B_{a}}{B}+\frac{A_{a}}{A}\right) \dot{a}^{2}+\frac{A_{a}}{A B}}{\sqrt{\Delta}}+\frac{\sqrt{\Delta} C_{a}}{C} .
\end{aligned}
$$

\section{Positive matter sources}

The energy-momentum components at the equilibrium condition, i.e. $a=a_{0}=$ constant with $\dot{a}=\ddot{a}=0$, yield

$$
\begin{aligned}
\sigma_{0} & =-\left.\left(\frac{C_{a}}{C}+\frac{F_{a}}{F}\right) \frac{1}{\sqrt{B}}\right|_{a=a_{0}} \\
P_{y 0} & =\left.\left(\frac{A_{a}}{A}+\frac{F_{a}}{F}\right) \frac{1}{\sqrt{B}}\right|_{a=a_{0}}, \\
P_{\phi 0} & =\left.\left(\frac{A_{a}}{A}+\frac{C_{a}}{C}\right) \frac{1}{\sqrt{B}}\right|_{a=a_{0}} .
\end{aligned}
$$




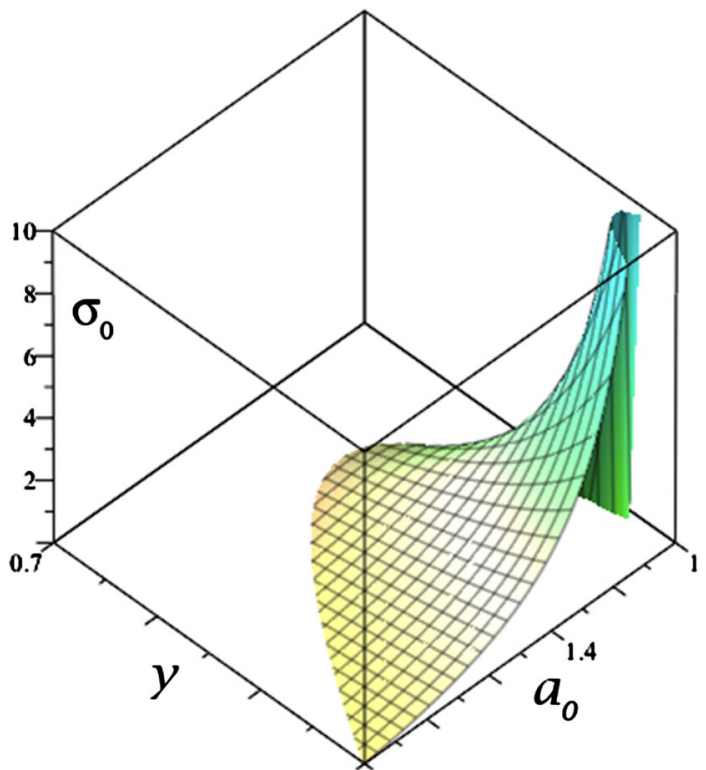

Fig. 1 A 3D plot of the positive part of $\sigma_{0}$ in terms of $a_{0}$ and $y$ with $\delta=2.0$. We see that, although $\sigma_{0}$ gets positive values for some interval of $y$, it is not positive everywhere on $y$. When the value of $\delta$ decreases, the interval of $y$ on which $\delta$ is positive gets smaller and ultimately for $\delta \leq 1$ the interval disappears so that $\sigma_{0}$ gets only negative values. (Note that $\sigma_{0}$ is an even function with respect to $y$ and only a section has been plotted.)

The explicit form of the energy-momentum components is then found to be

$$
\begin{aligned}
\sigma_{0} & =\frac{2\left[2 \delta\left(a_{0}^{2}-y^{2}\right)-2 a_{0}^{3}+a_{0}\left(1+y^{2}\right)+a_{0} \delta^{2}\left(y^{2}-1\right)\right]}{\left(a_{0}^{2}-1\right)\left(a_{0}^{2}-y^{2}\right)}, \\
P_{y 0} & =\frac{2 a_{0}}{a_{0}^{2}-1}, \\
P_{\phi 0} & =\frac{2 a_{0}\left[a_{0}^{2}-1+\left(1-y^{2}\right) \delta^{2}\right]}{\left(a_{0}^{2}-1\right)\left(a_{0}^{2}-y^{2}\right)} .
\end{aligned}
$$

Note that since $1<a_{0}$ there are no singularities in the foregoing expressions. From the conditions $a_{0}>1$ and $y<1$ one observes that $P_{y 0}$ and $P_{\phi 0}$ are both positive, while $\sigma_{0}$ may be positive, negative or zero. Figure 1 displays $\sigma_{0}$ in terms of $a_{0}$ and $y$. We observe that for $\delta>1$ there exist regions where $\sigma_{0}$ becomes positive. This is seen clearly in Fig. 1. Also in the interval on which $\sigma_{0} \geq 0$ the weak and strong energy conditions are satisfied.

We note that in Fig. 1 the energy density $\sigma_{0}$ is shown in terms of $y$ and $a_{0}$ but only $y$ is variable and $a_{0}$ is fixed for a specific TSW. This means that at the throat $x=a_{0}$, and only $y$ and $\phi$ are variable. As one sees from (18), there is an angular symmetry and as a result, not only $\sigma_{0}$ but also $P_{y 0}$ and $P_{\phi 0}$ are only functions of $y$. In addition, $P_{y 0}$ is a constant function of $y$. Therefore once we set the radius of the throat, i.e., $a_{0}$, our energy-momentum tensor's components are left with the only variable $y$. Hence, depending on $y$, the energy

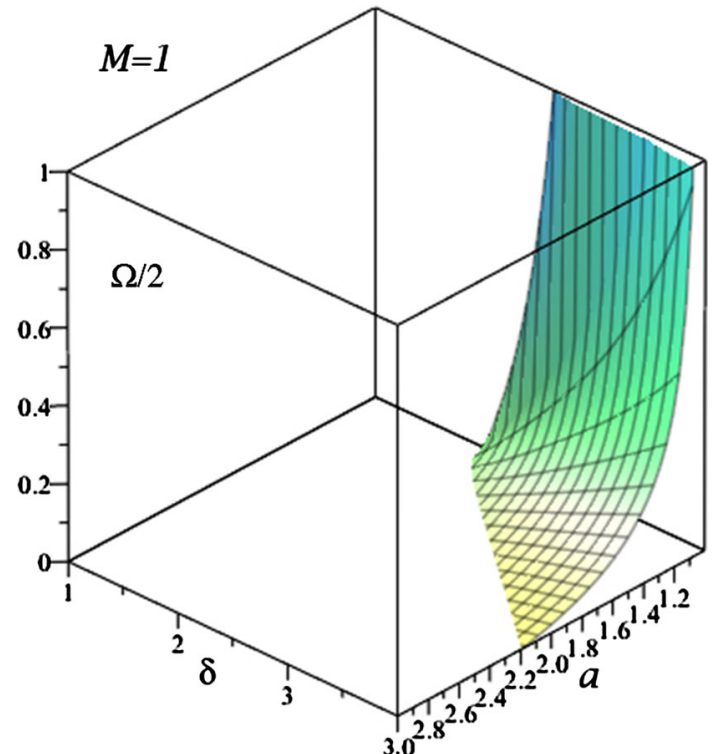

Fig. 2 A 3D plot of the positive amount of energy $\frac{\Omega}{2}$ versus $a$ and $\delta$ with constant mass parameter i.e. $M=1$. This figure shows clearly that for $\delta>2$ there exists $a_{c}$ in which, with $1<a<a_{c}$, the total energy is positive and therefore the resultant thin-shell wormhole is supported by ordinary/normal matter. It should be added that for a given $\delta>2$, there exists $1<a<a_{c}$, which leads to a physically acceptable TSW

density of the TSW i.e. $\sigma_{0}$ is locally positive or negative. This is what we see in Fig. 1. Of course, the situation is completely different for $P_{y 0}$ and $P_{\phi 0}$, which are positive for the entire domain of $y$.

In addition to the energy conditions we are mainly interested in the total energy supporting the TSW given by

$\Omega=2 \int_{0}^{2 \pi} \int_{0}^{1} \int_{1}^{\infty} \sigma_{0} \delta\left(x-a_{0}\right) \sqrt{-g} \mathrm{~d} x \mathrm{~d} y \mathrm{~d} \phi$,

which simplifies to

$\Omega=4 \pi \int_{0}^{1} \sigma_{0} \sqrt{-g_{0}} \mathrm{~d} y$.

In Eq. (19), $\delta\left(x-a_{0}\right)$ is the Dirac delta function. In Fig. 2 we plot $\Omega$ versus $a$ and $\delta$ with fixed value of mass $M=1$. Figure 3 reveals more details. These plots overall show that TSW supported by normal matter is possible provided $\delta>2$. That explains also why, given ordinary matter alone in Schwarzschild spacetime with $\delta=1$, there was no such traversable wormhole. In this regard let us add that even an arbitrarily small energy condition violation is considered significant [57].

\section{Stability analysis}

In this section we apply the small velocity perturbation on the shell under the condition that the EoS of the TSW after the 


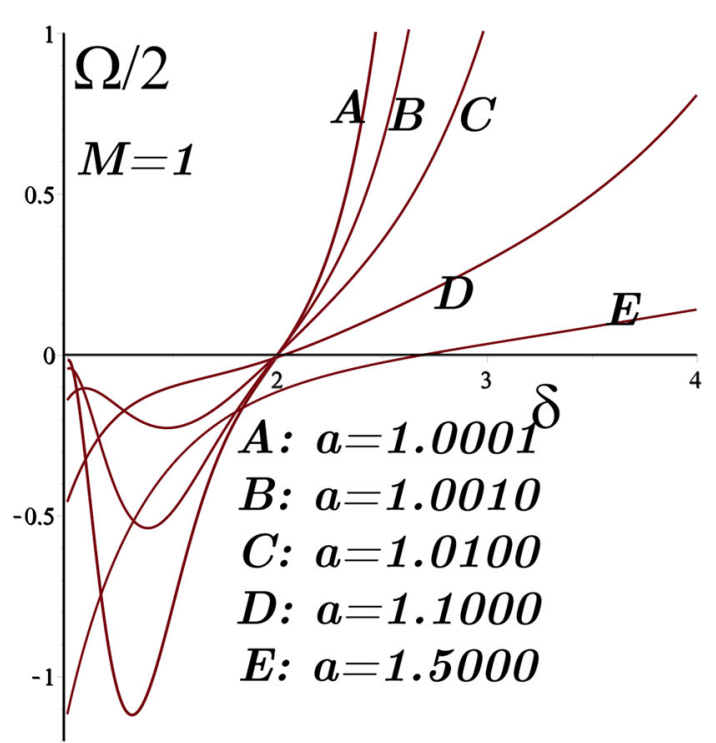

Fig. $3 \frac{\Omega}{2}$ versus $\delta$ for different values of $a=1.0001,1.0010$, $1.0100,1.1000,1.5000$, and $M=1$. As we commented in Fig. 2, here it is clearer that, when the value of $a \rightarrow 1$, the threshold $\delta$ which admits a positive total energy approaching $\delta=2$. This means that $\delta=2$ is a critical value as regards to have a thin-shell wormhole supported by ordinary matter. For larger $a$ the threshold $\delta$ gets larger values

perturbation is the same as its EoS at its static equilibrium. This is possible if the perturbation process occurs slowly enough, in which all the intermediate states can also be considered as static equilibrium points. Therefore the EoS after the perturbation reads

$\frac{P_{y}}{\sigma}=-\frac{\frac{A_{a}}{A}+\frac{F_{a}}{F}}{\frac{C_{a}}{C}+\frac{F_{a}}{F}}$

and

$$
\frac{P_{\varphi}}{\sigma}=-\frac{\frac{A_{a}}{A}+\frac{C_{a}}{C}}{\frac{C_{a}}{C}+\frac{F_{a}}{F}} .
$$

These in explicit form amount to

$2 \ddot{a}+\left(\frac{B_{a}}{B}\right) \dot{a}^{2}=0$,

which upon integration yields

$\dot{a}=\dot{a}_{0} \sqrt{\frac{B_{0}}{B}}=\dot{a}_{0} \sqrt{\frac{\left(\frac{a_{0}+1}{a_{0}-1}\right)^{\delta}\left(\frac{a_{0}^{2}-1}{a_{0}^{2}-y^{2}}\right)^{\left(\delta^{2}-1\right)}}{\left(\frac{a+1}{a-1}\right)^{\delta}\left(\frac{a^{2}-1}{a^{2}-y^{2}}\right)^{\left(\delta^{2}-1\right)}} .}$

In Figs. 4 and 5 we plot $\dot{a}$ in terms of $a$ and $y$ for the initial values $a_{0}=1.2$ and $\dot{a}_{0}= \pm 0.1$, respectively, and $\delta=2.5$. As one can see in both cases the velocity does not go to zero, which means that the throat does not go back to its initial position.

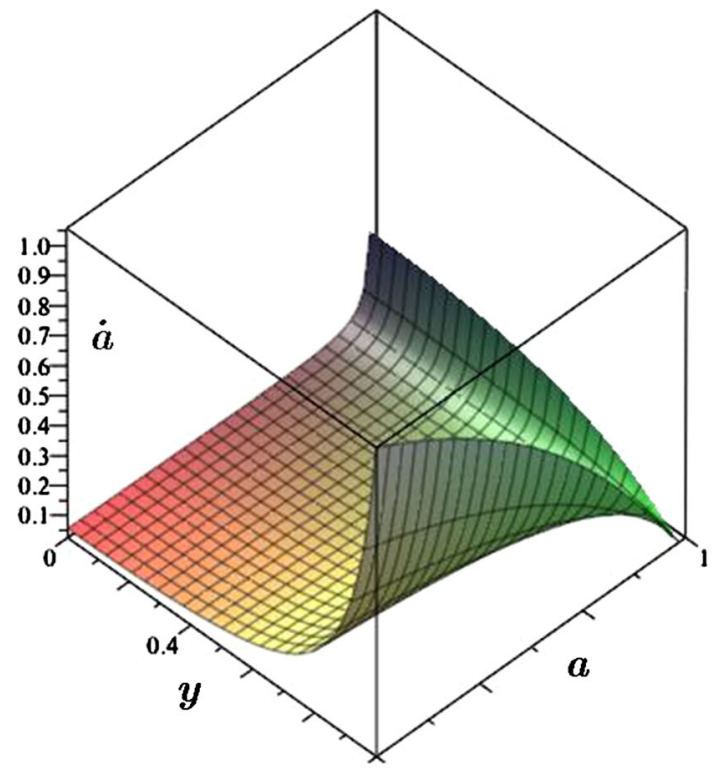

Fig. $4 \dot{a}$ versus $a$ and $y$ with $\delta=2.5, \dot{a}_{0}=0.1$ and $a_{0}=1.2$. The velocity never vanishes, which is an indication of instability of the throat under a small velocity perturbation

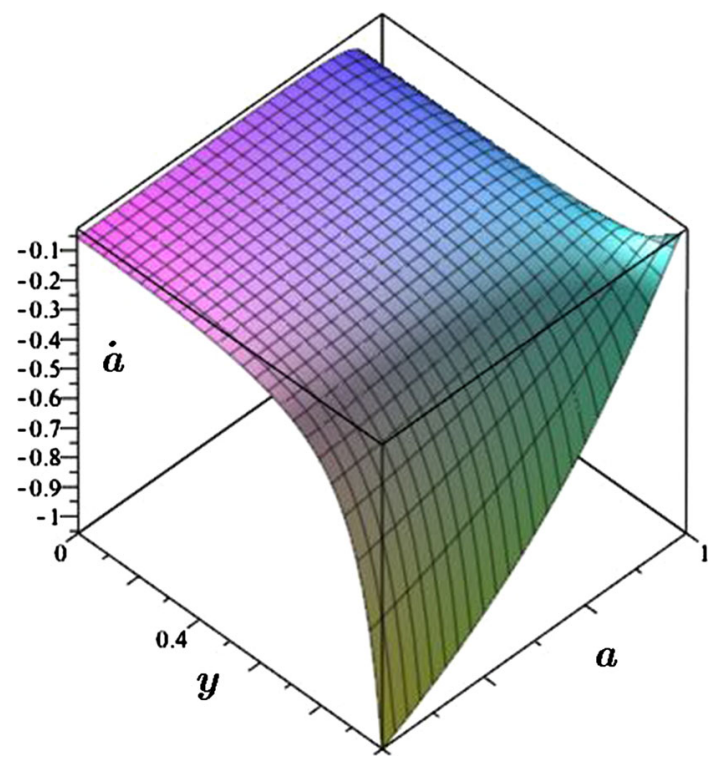

Fig. 5 A $3 \mathrm{D}$ plot of $\dot{a}$ versus $a$ and $y$ with $\delta=2.5, \dot{a}_{0}=-0.1$ and $a_{0}=1.2$. The velocity fails to vanish, which amounts to instability of the throat under a small velocity perturbation

\section{Conclusion}

We considered the possibility of having a physical wormhole solution in Einstein's general relativity, which is supported by normal matter and where the energy conditions are satisfied. Our emphasis is on Einstein's general relativity instead of modified theories such as the Lovelock theory. In such theories there were attempts to find such physical wormholes [37-44] with a non-physical solution and it was shown that a 
TSW supported by positive energy was possible. In this context we have shown that TSWs with oblate sources can be employed to admit overall physical (i.e. non-exotic) matter even in Einstein's general relativity. As we have depicted in the figures, for $\delta>2$ not only the total energy of the wormhole is positive but also the WECs and SECs are satisfied for a limited $y$-interval, which increases for larger $\delta$. A certain range for the deviation from spherical symmetry can be chosen from the total energy integral to render this possible. Locally it can easily be checked from Eq. (16) for $y=0$, for instance, that we have a negative energy density; however, this is compensated within the total energy expression. It is also expected that, once the metric and the surface energymomentum become time dependent, energy conservation on the thin shell will not be valid any more. Another important aspect concerning TSWs which has not been discussed here is their stability against perturbations. It is shown that small velocity perturbations in the $x$-direction leads to an unstable wormhole throat. Finally, out of curiosity we wish to ask: does the deformation parameter $\delta$ save wormholes other than TSWs in Einstein's theory? this remains to be seen.

Open Access This article is distributed under the terms of the Creative Commons Attribution License which permits any use, distribution, and reproduction in any medium, provided the original author(s) and the source are credited.

Funded by $\mathrm{SCOAP}^{3}$ / License Version CC BY 4.0.

\section{References}

1. M.S. Morris, K.S. Thorne, Am. J. Phys. 56, 5 (1998)

2. A. Einstein, N. Rosen, Phys. Rev. 48, 73 (1935)

3. M. Visser, Lorentzian Wormholes: From Einstein to Hawking (American Institute of Physics, New York, 1995)

4. M. Visser, Phys. Rev. D 39, 3182 (1989)

5. M. Visser, Nucl. Phys. B 328, 203 (1989)

6. E. Poisson, M. Visser, Phys. Rev. D 52, 7318 (1995)

7. P.R. Brady, J. Louko, E. Poisson, Phys. Rev. D 44, 1891 (1991)

8. M. Ishak, K. Lake, Phys. Rev. D 65, 044011 (2002)

9. E.F. Eiroa, C. Simeone, Phys. Rev. D 70, 044008 (2004)

10. E.F. Eiroa, C. Simeone, Phys. Rev. D 81, 084022 (2010)

11. E.F. Eiroa, C. Simeone, Phys. Rev. D 71, 127501 (2005)

12. E.F. Eiroa, Phys. Rev. D 78, 024018 (2008)

13. E.F. Eiroa, G.F. Aguirre, Eur. Phys. J. C 72, 2240 (2012)

14. C. Bejarano, E.F. Eiroa, Phys. Rev. D 84, 064043 (2011)

15. E.F. Eiroa, C. Simeone, Phys. Rev. D 76, 024021 (2007)
16. E.F. Eiroa, Phys. Rev. D 80, 044033 (2009)

17. M.G. Richarte, Phys. Rev. D 82, 044021 (2010)

18. E.F. Eiroa, C. Simeone, Phys. Rev. D 82, 084039 (2010)

19. F.S.N. Lobo, Phys. Rev. D 71, 124022 (2005)

20. N.M. Garcia, F.S.N. Lobo, M. Visser, Phys. Rev. D 86, 044026 (2012)

21. J.P.S. Lemos, F.S.N. Lobo, Phys. Rev. D 78, 044030 (2008)

22. M.H. Dehghani, M.R. Mehdizadeh, Phys. Rev. D 85, 024024 (2012)

23. M. Sharif, M. Azam, JCAP 04, 023 (2013)

24. M. Sharif, M. Azam, JCAP 05, 25 (2013)

25. M. Sharif, M. Azam, Eur. Phys. J. C 73, 2407 (2013)

26. M. Sharif, M. Azam, Eur. Phys. J. C 73, 2554 (2013)

27. M. Jamil, M.U. Farooq, M.A. Rashid, Eur. Phys. J. C 59, 907 (2009)

28. H. Weyl, Ann. Phys. 54, 117 (1917)

29. D.M. Zipoy, J. Math. Phys. (N.Y.) 7, 1137 (1966)

30. B.H. Voorhees, Phys. Rev. D 2, 2119 (1970)

31. G.L. Gerakopoulos, Phys. Rev. D 86, 044013 (2012)

32. N.A. Collins, S.A. Hughes, Phys. Rev. D 69, 124022 (2004)

33. A. Tomimatsu, H. Sato, Phys. Rev. Lett. 29, 1344 (1972)

34. A. Tomimatsu, H. Sato, Prog. Theor. Phys. 50, 95 (1973)

35. R.M. Kerns, W.J. Wild, Phys. Rev. D 26, 3726 (1982)

36. M. Halilsoy, J. Math. Phys. 33, 4225 (1992)

37. M.G. Richarte, C. Simeone, Phys. Rev. D 76, 087502 (2007)

38. M.G. Richarte, C. Simeone, Phys. Rev. D 77, 089903(E) (2008)

39. C. Simeone, Phys. Rev. D 83, 087503 (2011)

40. S.H. Mazharimousavi, M. Halilsoy, Z. Amirabi, Phys. Rev. D 81, 104002 (2010)

41. S.H. Mazharimousavi, M. Halilsoy, Z. Amirabi, Class. Quantum Gravity 28, 025004 (2011)

42. T. Bandyophyay, S. Chakraborty, Class. Quantum Gravity 26, 085005 (2009)

43. P. Kanti, B. Kleihaus, J. Kunz, Phys. Rev. Lett. 107, 271101 (2011)

44. P. Kanti, B. Kleihaus, J. Kunz, Phys. Rev. D 85, 044007 (2012)

45. O. Obregón, H. Quevedo, M.P. Ryan, JHEP 07, 005 (2004)

46. O. Obregón, H. Quevedo, M.P. Ryan, Phys. Rev. D 70, 064035 (2004)

47. L. Herrera, F.M. Paiva, N.O. Santos, J. Math. Phys. (N.Y.) 40, 4064 (1999)

48. H. Kodama, W. Hikida, Class. Quantum Gravity 20, 5121 (2003)

49. D. Papadopoulos, B. Stewart, L. Witten, Phys. Rev. D 24, 320 (1981)

50. D. Hochberg, M. Visser, Phys. Rev. D 56, 4745 (1997)

51. S.H. Mazharimousavi, M. Halilsoy, Flare-out conditions in static thin-shell wormholes. (2014). arXiv:1311.6697

52. W. Israel, Nuovo Cimento 44B, 1 (1966)

53. V. de la Cruzand, W. Israel, Nuovo Cimento 51A, 774 (1967)

54. J.E. Chase, Nuovo Cimento 67B, 136 (1970)

55. S.K. Blau, E.I. Guendelman, A.H. Guth, Phys. Rev. D 35, 1747 (1987)

56. R. Balbinot, E. Poisson, Phys. Rev. D 41, 395 (1990)

57. M. Visser, S. Kar, N. Dadhich, Phys. Rev. Lett. 90, 201102 (2003) 